\title{
Reliability Evaluation of Distribution Power Systems Based on Artificial Neural Network Techniques
}

\author{
Mohammoud M. Hadow, ${ }^{1}$ Ahmed N. Abd Allah, ${ }^{1}$ and Sazali P. Abdul karim ${ }^{2}$ \\ ${ }^{1}$ Faculty of Electrical and Electronics Engineering, University of Malaysia, Pahang, Lebuhraya Tun Razak, \\ Kuantan Pahang, 26300 Gambang, Malaysia \\ ${ }^{2}$ National Company, Engineering, Transmission Division TNB, Kuala Lumpur, Malaysia
}

Correspondence should be addressed to Mohammoud M. Hadow, hadow50@yahoo.com

Received 5 May 2011; Revised 21 July 2011; Accepted 25 August 2011

Academic Editor: Edgar Carreno

Copyright (C) 2012 Mohammoud M. Hadow et al. This is an open access article distributed under the Creative Commons Attribution License, which permits unrestricted use, distribution, and reproduction in any medium, provided the original work is properly cited.

\begin{abstract}
In order to assess the reliability of distribution systems, more and more researchers are directing their attention to the artificial intelligent method, and several reliability indices have been proposed, such as basic load point indices and system performance indices. Artificial neural network is recently established as a useful and much promising too, applied to variety of power systems engineering. This paper presents ANN version for evaluating the reliability of distribution power systems (DPSs), in the proposed algorithm, the ANN used to predicted (RPS) using historical data method constructed according to the backpropagation learning rule. At the same time, System indices such as SAIFI and SAIDI of real distribution system are computed and compared with results generated by network method. The result obtained by proposed method gives acceptable reliability indices and can also found that the deviation of computed values by the proposed method is less than $1 \%$ and needs running time on ASUN network environment of less than $2 \mathrm{~s}$. The ANN approach demonstrates advantage over the network method.
\end{abstract}

\section{Introduction}

Broadly speaking, electrical power distribution systems include all parts of electrical utility systems between bulk power sources and the consumers' service-entrance equipment. The main function of distribution network is to supply electrical power generated from large sources to consumers at desired voltage level and with a degree of appropriate reliability [1]. Reliability and efficient maintenance are crucial for the continuity of electrical energy. It is customary for moderate distribution networks to have a large number of nodes, to serve a vast geographical area and to ensure a safe operation at severe ambient conditions [2]. Distribution networks operating at several voltage levels including the networks of local or municipal unities are the primary parts of view; therefore, distribution system reliability evaluation is major preoccupation of the power company, in particular, in face of emergency and necessary reconfiguration.

The techniques used in power system reliability evaluation can be divided into the two basic categories of analytical and simulation methods. The analytical techniques are highly developed and have been used in practical applications for several decades [3]. Several methods [1-5] have been presented in literature for evaluating distribution system reliability; They have been divided in two abroad groups, firstly based on solution of logic networks and secondly on the solution of state-space models. Between them, these methods can accommodate most practical systems. In addition, the network methods for determining system reliability are comparatively simple and direct.

In this paper, it is proposed to solve the problem of distribution system reliability evaluation by ANN which can learn from patterns encountered previously. Many types of network exist [1-8], but this study described here focuses on three layer feed forward network with backpropagation learning rule [4]; the developed ANN was utilized to evaluate the distribution system reliability. Three different cases are studied on a real distribution system in (north Taiwan); the output of the result from the developed ANN is satisfactory compared with that computed by network method with sufficient learning, the ANN generates result very fast and incurs only tiny deviation. The ANN also has an advantage over 
the network method in computing system reliability when one or more circuit breaker opens due to a fault or overhead switching. Under such situation, the network method repeats the whole computation procedure because the system logic diagram differs. Yet in the ANN approach the related links are simply modified by resetting their weights.

The paper organized as follows: section proposed methodology includes ANN approach Section 3 test systems for the case study and Section 4 where result and discussion and Section 5 are concludes the paper.

\section{The Proposed Methodology}

2.1. Artificial Neural Network (ANN) Approach. Among the various kinds of ANN approaches that exit, the backpropagation (BP) learning algorithm, which has become the most popular in engineering applications, was used in this study. This network has three layers, one input layer, one hidden layer, and one output layer. To train and test the neural networks, input data patterns and corresponding targets were required. In developing a ANN model, the available data set was divided into two sets, one to be used for training of the network (70-80\% of the data), and the remaining was used to verify the generalization capability of the network. The mathematical background, the procedures for training and testing the ANN, and an account of its history can be found in the text by Haykin [6]. Input-output pairs are presented to the network, and weights are adjusted to minimize the error between the network output and actual value. Once training is completed, predictions from a new set of data may be done using the already trained network.

The Neural Networks Toolbox of MATLAB 5.2 was used to form the ANN. The log-sigmoid transfer function was used in the hidden layer (first layer) and output layer (second layer). Inputs of system determine the neuron number in the input layer of the network, and its outputs determine the neuron number in the output layer of the network. Thus, input layer of network has three neurons and the output layer has two neurons. Seven neurons were used in hidden layer. Neural network requires that the range of both the input and output values should be between 0.1 and 0.9 . The following formula is used:

$$
\frac{\text { Actual Value }- \text { Minimum }}{\text { Maximum }- \text { Minimum }} *(\text { high }- \text { low })+\text { low }
$$

This equation is a widely employed method in unification $[4,5]$, where minimum is minimum data value, maximum is the maximum data value, high is the maximum normalized data which equals 0.9 , and low is the minimum normalized data which equals 0.1 .

The backpropagation network training function updates weight and bias values according to Leven-berg-Marquardt optimization. The Levenberg-Marquardt algorithm is very well suited to neural network training, where the performance index is the mean squared error [14]. Mean squared error (MSE) that determines network performance is formulated as follows in (2). The backpropagations learning rule is an iterative gradient algorithm designed to minimize the mean square error between the actual output of multilayer feed forward network and desired output:

$$
\text { MSE }=\frac{1}{N} \sum_{i=1}^{N}\left(y_{i}-y_{k}\right)^{2},
$$

where, $y_{i}$ is the predicted value of the $i$ th pattern, $y_{k}$ is the target value of the $i$ th pattern, and $N$ is the number of pattern.

An essential component of the rule is the iterative method that propagates error term required to adapted weights back from nodes in the output layer to needs in the lower layer. At the beginning, all weights and node offsets are set to small random values. The input values are presented, and desired outputs are specified. Then, the network is used to calculate actual output. As recursive algorithm, starting at the output nodes and working back to hidden layer, adjusts weights until they converge and the objective function is reduced to acceptable value. The training was repeated by presenting different sets of input data to the ANN.

\section{Test Systems for the Case Study}

3.1. Test System. A range of reliability indices were calculated for a number of studies. The methods for evaluating these indices are described in detail in [2] and applied to practical systems in [8]. The indices include load point indices. These are failure rate $(A)$, outage time $(r)$, annual unavailability $(U)$, load disconnected $(L)$, and energy not supplied $(E)$. These can be system indices. These are SAIFI, SAIDI, CAIDI, ASAI, ASUI, ENS, and AENS. They are fully specified and defined in [2] and can be evaluated from the load point indices for a group of load points or the whole system. The studies performed include llkV feeders. These studies consider the $1 \mathrm{kV}$ feeders only and ignore any failures in the $33 \mathrm{kV}$ system, the $33 / 11 \mathrm{kV}$ substation, and the $1 \mathrm{kV}$ breakers. They assume that the IlkV source breaker operates successfully when required, disconnects are opened whenever possible to isolate a fault, and the supply restored to as many load points as possible using appropriate disconnects and the alternative supply if available is $33 \mathrm{kV}$ system. These studies evaluate the reliability indices at the $1 \mathrm{kV}$ supply point busbars. They ignore any failures on the incoming $33 \mathrm{kV}$ supply circuits. They include the effect of passive and active failures [2] on all components from the $33 \mathrm{kV}$ busbars down to the $1 \mathrm{kV}$ supply point busbars together with active failures on the outgoing $11 \mathrm{kV}$ feeder breakers, This study system comprises one substation and feeders. As show in Figure 1, the substation is labeled X and feeders are labeled X1, X2, X3,.., X30 and the feeder load is show in Table 1.

3.2. The Reliability Indices. This index can then be combined with the customer composition in the distribution system to evaluate the system indices of SAIFI, SAIDI, CAIDI, ASAI, and so forth [7]. In the distribution area, the usual indices are the load point failure rate (or frequency), the average outage duration, and the average annual outage time. Normal utility practise is to measure distribution system performance in terms of SAIFI, SAIDI. This approach does not usually 
TABLE 1: Load and customer data for each Feeder.

\begin{tabular}{lcc}
\hline Feeder & Load $(\mathrm{kVA})$ & No. of customers \\
\hline 11 & 48642.00 & 216 \\
12 & 5418.7 & 3277 \\
13 & 8470.25 & 6783 \\
14 & 7376.5 & 3344 \\
15 & 1222.5 & 86 \\
16 & 3240.5 & 229 \\
17 & 6280.5 & 495 \\
18 & 2606.00 & 175 \\
19 & 4247.25 & 384 \\
20 & 4107.00 & 649 \\
21 & 3706.5 & 1132 \\
22 & 5418.50 & 3277 \\
\hline
\end{tabular}

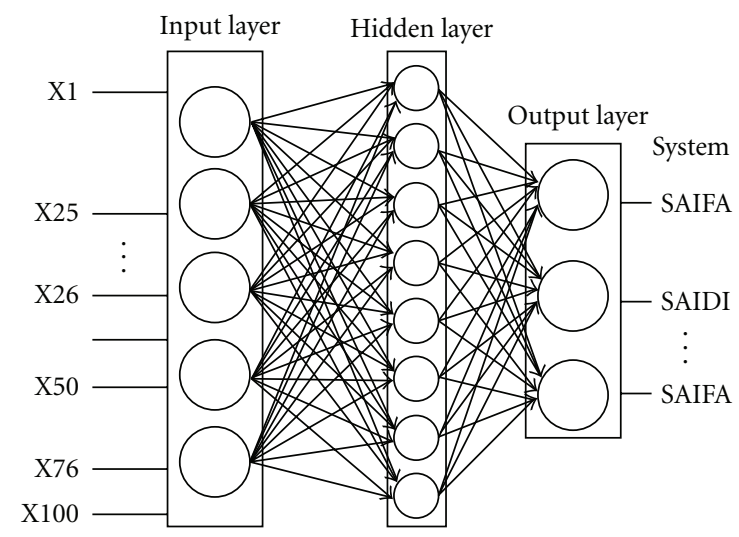

FIGURE 1: Structure of neural network.

encompass any discussion or appreciation that the customerbased indices can exhibit considerable natural variability on an annual basis, while still having the expected values determined by the analytical approach. In order to obtain this appreciation of the annual variability, it is necessary to include probability or frequency distribution concepts in the evaluation process to evaluate reliability of test distribution system; the following data are needed: (1) the load at each point, (2) the system configuration, (3) numbers of customer at each load point, (4) averge failure rate $(\lambda)$ of all component such as lines, transformers, breakers and so forth, (6) the restoration time $\mathrm{r}$ of each component, and (7) the switches times s of switches and breakers. With these data available at hand, the following two reliability indices are computed [57]: the system average interruption frequency index (SAIFI) and system average interruption duration index as following in (3) and (4):

$$
\begin{gathered}
\text { SAIFI }=\frac{\text { Total number of customer interruption }}{\text { Total number of customer severed }}, \\
\text { SAIFI }=\frac{\sum \lambda_{i} N_{i}}{\sum N_{i}},
\end{gathered}
$$

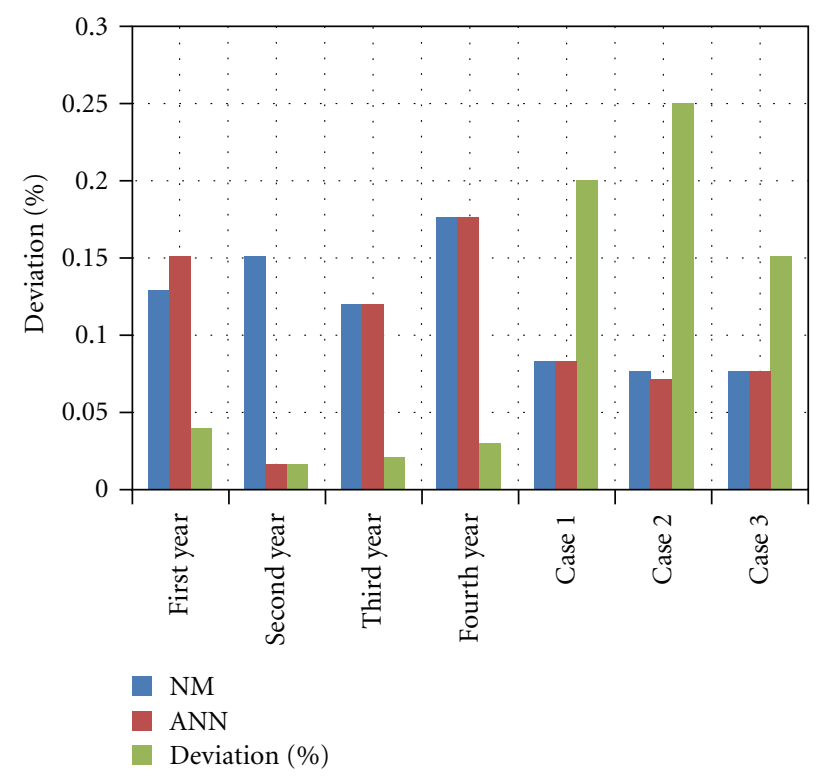

FIGURE 2: SAIFI values feeder X14 with deviation less than 0.25.

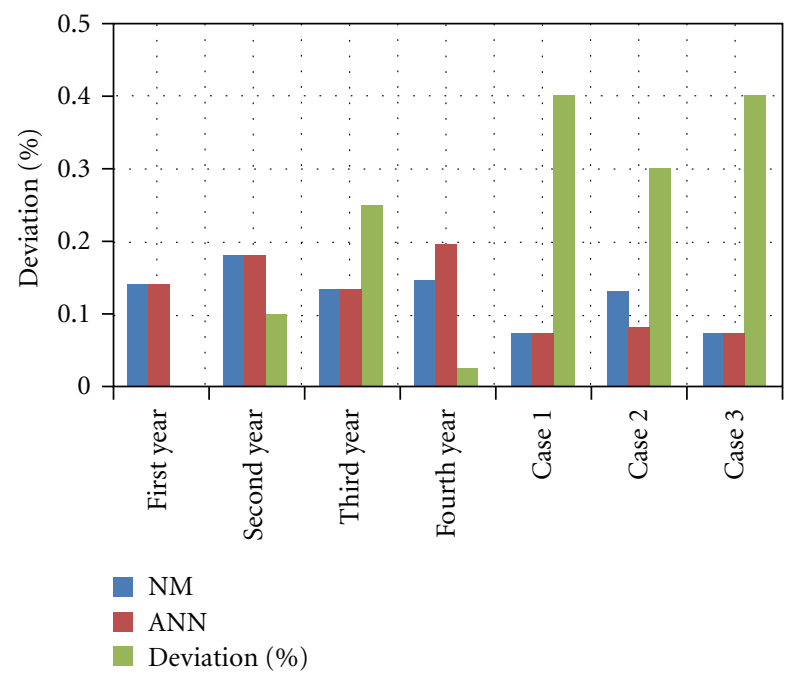

FIgURe 3: SAIFI values for feeder X12 with deviation less than 0.4.

where $\left(\lambda_{i}\right)$ is average failure rate and $N_{i}$ is number of customer at load point

$$
\begin{gathered}
\text { SAIDI }=\frac{\text { Sum of customer interruption duration }}{\text { Total number of customer severed }}, \\
\text { SAIFI }=\left(\frac{\sum U_{i} N_{i}}{\sum N_{i}}\right),
\end{gathered}
$$

where $U_{i}$ is annual outage time.

\section{Results and Discussion}

In this work, the historical data of the test system for four years were provided by the power company. Using this data, the following cases were designed to study the applicability of the ANN approach. Case one prediction of the reliability 
TABLE 2: System average interruption frequency index values (SAIFI).

\begin{tabular}{lccccccccc}
\hline & \multicolumn{3}{c}{ Feeder14 } & \multicolumn{3}{c}{ Feeder12 } & \multicolumn{3}{c}{ System } \\
& $(\mathrm{NM})$ & $(\mathrm{ANN})$ & Deviation $(\%)$ & $\mathrm{NM}$ & ANN & Deviations (\%) & NM & ANN & Deviation (\%) \\
\hline Fist year & 0.1292 & 0.1493 & 0.04 & 0.14425 & 0.14295 & 0.0 .25 & 0.3934 & 0.39345 & 0.005 \\
Second year & 0.14935 & 0.01493 & 0.015 & 0.18255 & 0.18125 & 0.10 & 0.45255 & 0.4526 & 0 \\
Third year & 0.11915 & 0.1191 & 0.02 & 0.137 & 0.1363 & 0.25 & 0.37085 & 0.32085 & 0 \\
Fourth year & 0.17565 & 0.17575 & 0.03 & 0.1478 & 0.1977 & 0.025 & 0.52075 & 0.52075 & 0 \\
Case 1 & 0.0827 & 0.08235 & 0.2 & 0.0733 & 0.0736 & 0.40 & 0.2524 & 0.2542 & 0 \\
Case 2 & 0.0758 & 0.07045 & 0.25 & 0.1324 & 0.0829 & 0.30 & 0.2524 & 0.2541 & 0.02 \\
Case 3 & 0.0763 & 0.07605 & 0.15 & 0.0733 & 0.0739 & 0.40 & 0.25405 & 0.2542 & 0.53 \\
\hline
\end{tabular}

TABLE 3: System average interruption duration index values (SAIDI).

\begin{tabular}{|c|c|c|c|c|c|c|c|c|c|}
\hline & \multicolumn{3}{|c|}{$\mathrm{X} 14$} & \multicolumn{3}{|c|}{$\mathrm{X} 12$} & \multicolumn{3}{|c|}{ System } \\
\hline & $(\mathrm{NM})$ & $(\mathrm{ANN})$ & Deviation (\%) & NM & ANN & Deviations (\%) & $\mathrm{NM}$ & ANN & Deviation (\%) \\
\hline First year & 0.1292 & 0.1293 & 0.04 & 0.1423 & 0.01429 & 0.30 & 0.3924 & 0.3935 & 0.03 \\
\hline Second year & 0.14935 & 0.1493 & 0.02 & 0.1822 & 0.1818 & 0.15 & 0.4526 & 0.4526 & 0.00 \\
\hline Third year & 0.1193 & 0.1191 & 0.02 & 0.1320 & 0.1363 & 0.25 & 0.3708 & 0.3708 & 0.00 \\
\hline Fourth year & 0.1757 & 0.1758 & 0.03 & 0.1973 & 0.1977 & 0.03 & 0.1 .0208 & 0.5206 & 0.00 \\
\hline Case 1 & 0.0827 & 0.0824 & 0.20 & 0.0733 & 0.0736 & 0.40 & 0.2524 & 0.2542 & 0.00 \\
\hline Case 2 & 0.0758 & 0.0754 & 0.25 & 0.0824 & 0.0826 & 0.30 & 0.2524 & 0.2541 & 0.02 \\
\hline Case 3 & 0.0713 & 0.0711 & 0.15 & 0.0733 & 0.0739 & 0.40 & 0.2542 & 0.2542 & 0.54 \\
\hline
\end{tabular}

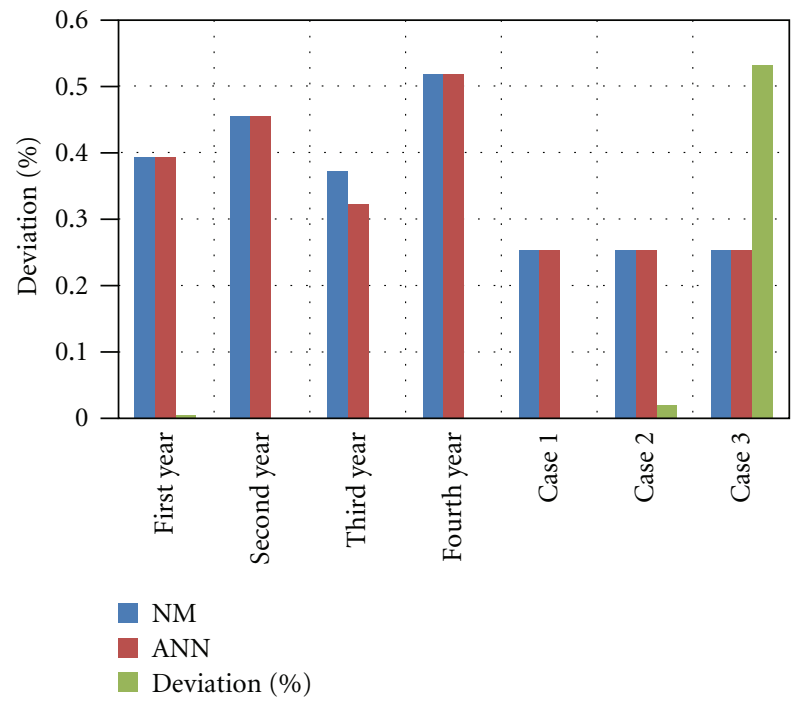

FIGURE 4: SAIFI values for feeder whole system with deviation less than 0.55 .

indices of 1996 by feeding the ANN with system data from 1996 to 1995 . Case 2. Evaluation of reliability indices when the feeder X14 is overload and part of its load is switched to X12. Case 3. Evaluation of the reliability indices when a fault occurs in feeder X14. The fault location is indicated by the arrow in Figure 1.

The developed ANN is shown in Figure 1. The data of feeder X11, X12,.., X22 enter the network from the input layer. The output layer result value of SAIFI, SAIDI of the whole system and each feeder. Corresponding to the number

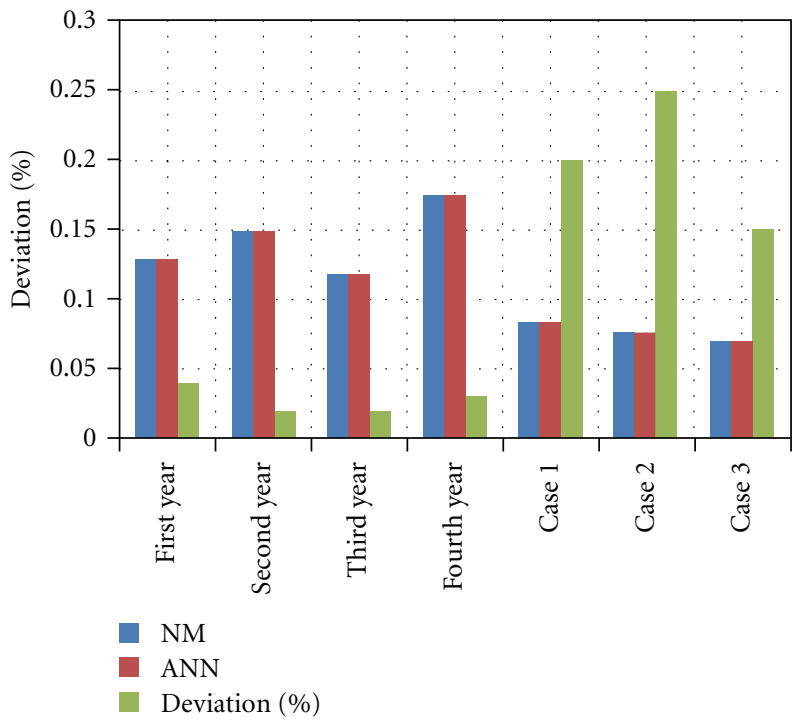

FIGURE 5: SAIDI values for feeder X14 with deviation less than 0.25.

of lines, buses, circuit breaker, and transformer of each feeder, this network contains 1809 nodes in the input layer, 18 nodes in output layer, and 100 nodes in the hidden layer.

The SAIFI, SAIDI values of X14, X12, and the whole system are given in Tables 2 and 3, respectively, the values computed by ANN are compared with results from the network method. The deviations are found in the Tables 2 and 3; also the deviation is illustrated in Figures 2-7. it has found that the deviations of the values computed by the proposed approach from those network methods are less than 0.005 . 


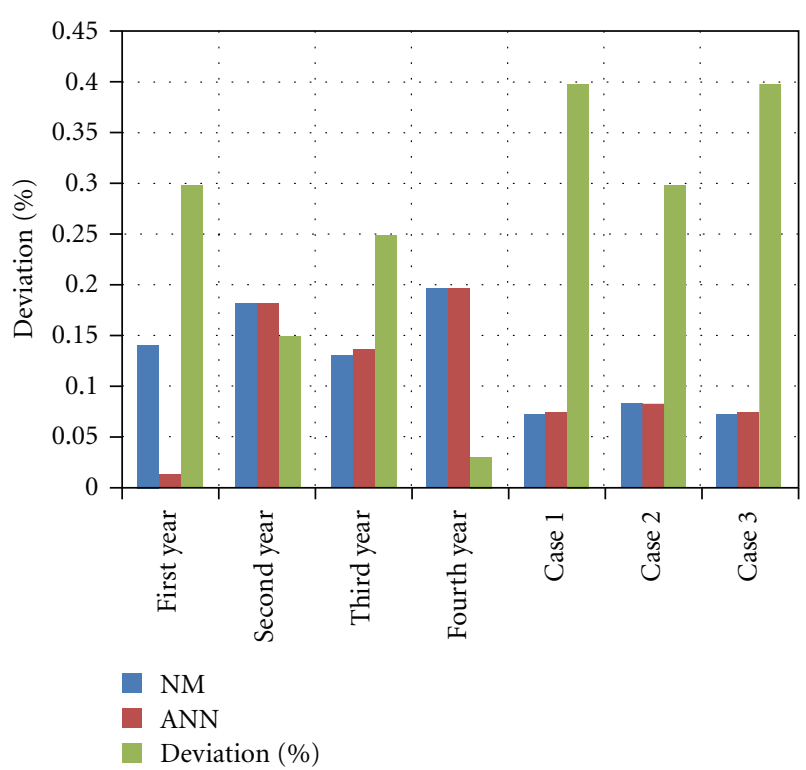

FIGURE 6: SAIDI values for feeder X12 with deviation less than 0.4.

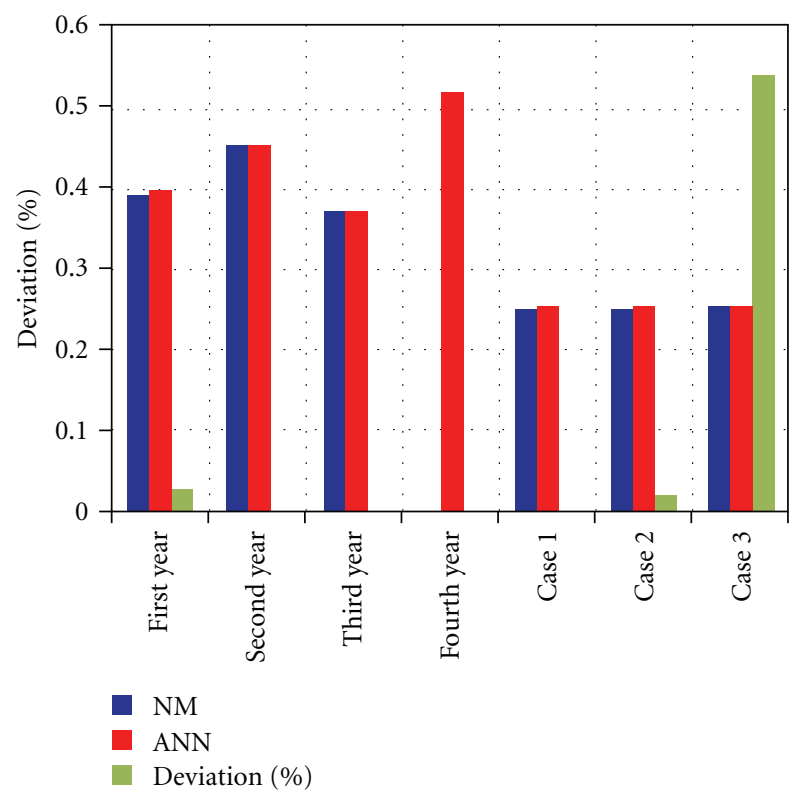

FIgURE 7: Total SAIDI values with deviation for whole system less than 0.54 .

The required running time on ASUN network environment is less than $2 \mathrm{~s}$, and the time required for the network method is 3.5 times greater than of ANN approach.

\section{Conclusions}

This paper has presented evaluating the reliability of distribution systems and backpropagation neural network model with a numbers of input layer-hidden layer-output layer constructed this neural network approach could be considered as alternative and practical techniqueing to evaluate distribution system indices. The cases were designed to demonstrate the capability of develop the neural network. With sufficient learning from historical data, the reliability indices of the normal, overload, and faulted conditions are computed with tiny deviations, below 0.005 from the results by the network method. The required computing times are less than $2 \mathrm{~s}$. Although ANN approach is generally time consuming if one wishes to develop the best configuration for training period, it is feasible due to its ability to learn and generalize a wide range of experimental conditions. This makes ANN a powerful tool to assist distribution system for solving complicated engineering problems.

\section{References}

[1] R. Billinton and W. li, Reliability Assessment of Power Systems Using Montecarlo Method, Plenum Press, New York, NY, USA, 1994.

[2] R. Billinton and R. N. Alan, Reliability Evaluation of Power Systems, Plenum Press, New York, NY, USA, 2nd edition, 1995.

[3] D. Midence, S. Rivera, and A. Vargas, "Reliability assessment in power distribution networks by logical and matrix operations," in Proceedings of the IEEE/PES Transmission and Distribution Conference and Exposition, August 2008.

[4] I. A. Basheer and M. Hajmeer, "Artificial neural networks: fundamentals, computing, design, and application," Journal of Microbiological Methods, vol. 43, no. 1, pp. 3-31, 2000.

[5] G. E. Nasr, E. A. Badr, and C. Joun, "Backpropagation neural networks for modeling gasoline consumption," Energy Conversion and Management, vol. 44, no. 6, pp. 893-905, 2003.

[6] S. Haykin, Neural Networks, A Comprehensive Foundation, McMillian College Publishing Company, New York, NY, USA, 1994.

[7] C. L. Chen and J. L. Chen, "A neural network approach for evaluating distribution system reliability," Electric Power Systems Research, vol. 26, no. 3, pp. 225-229, 1993.

[8] W. M. Lin, T. S. Zhan, and C. D. Yang, "Distribution system reliability worth analysis with the customer cost model based on RBF neural network," IEEE Transactions on Power Delivery, vol. 18, no. 3, pp. 1015-1021, 2003. 

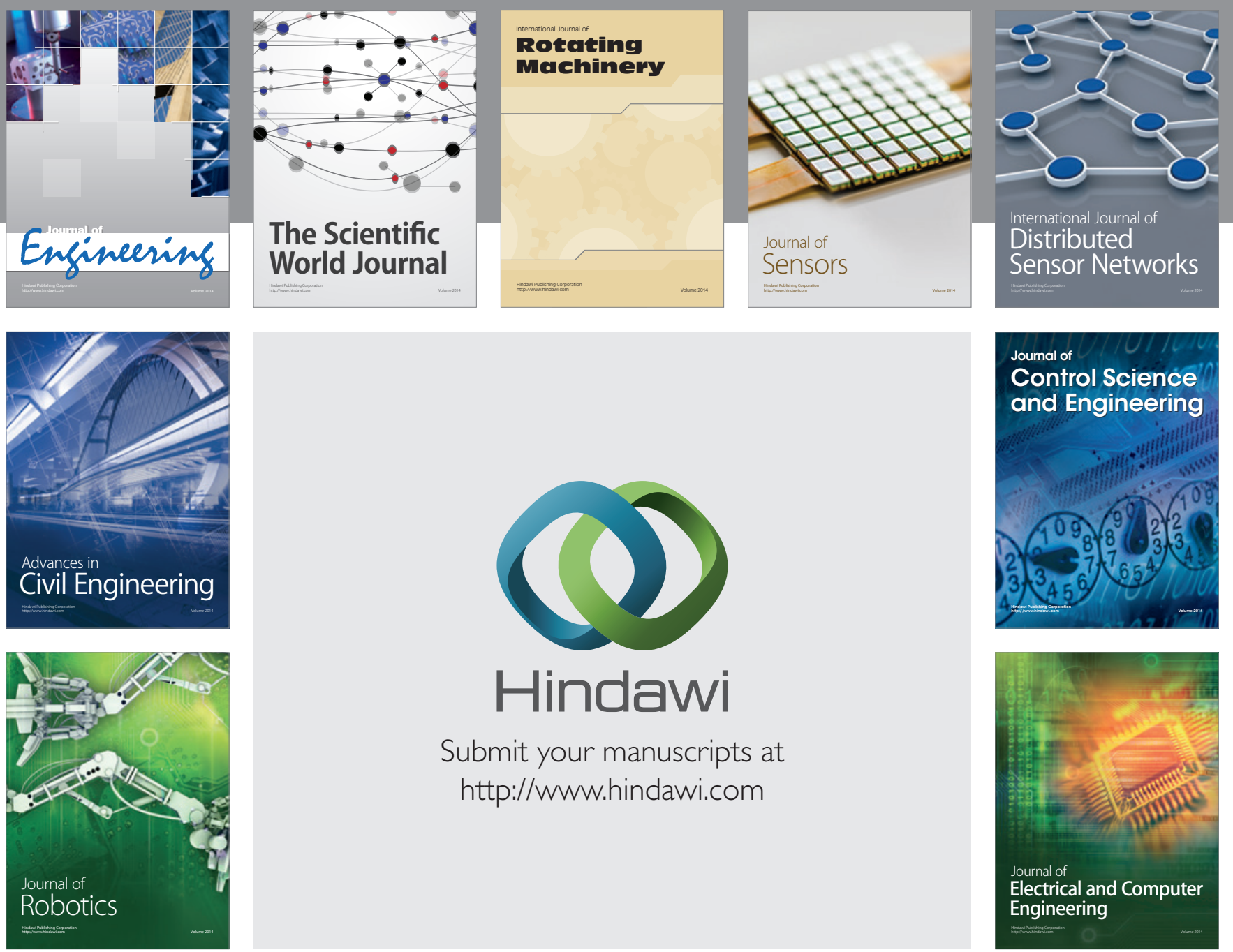

Submit your manuscripts at

http://www.hindawi.com
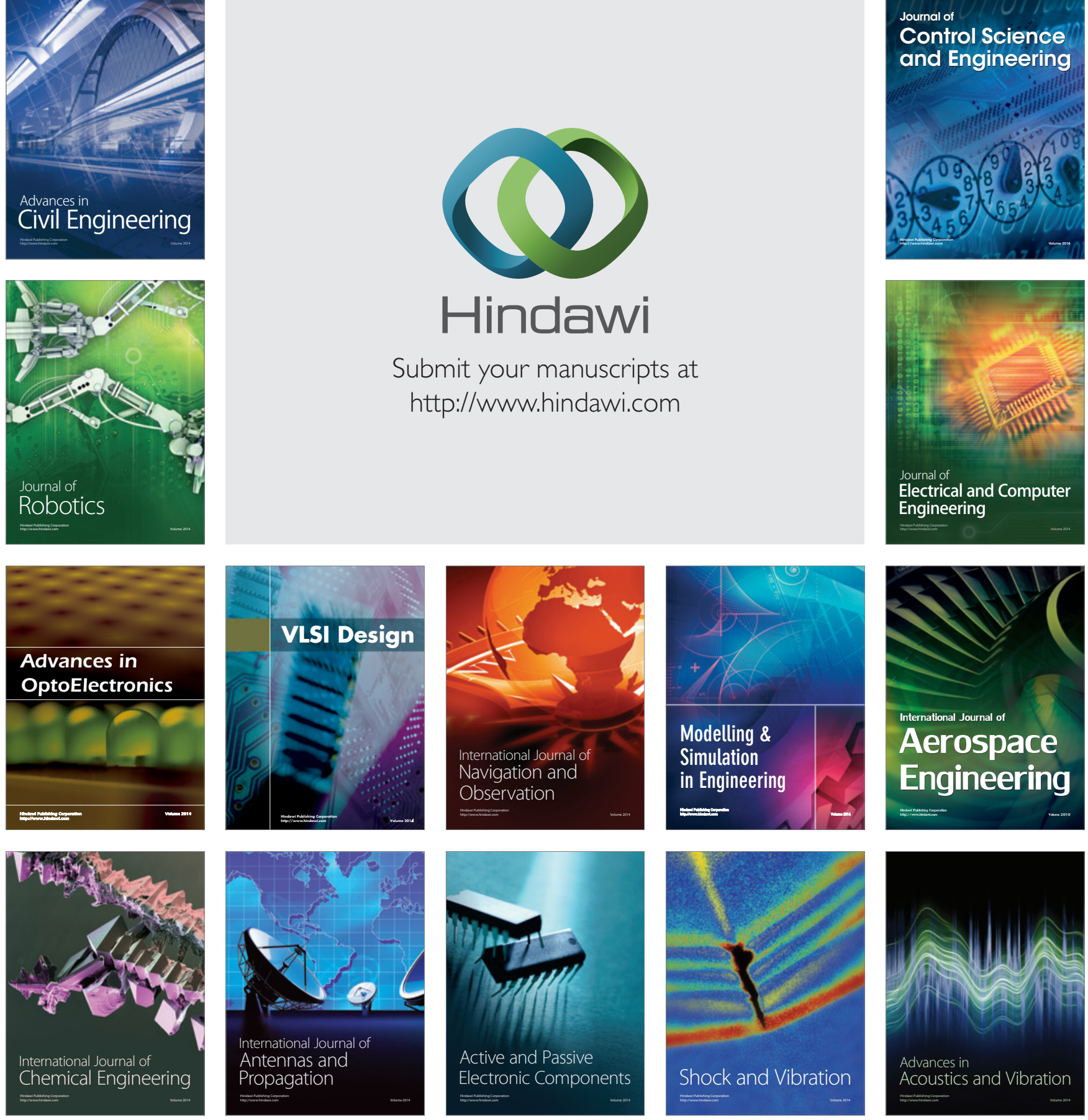